\title{
Pulmonary tuberculosis with multifocal skeletal involvement
}

\author{
A. Kaya ${ }^{1}$, Z. Topu ${ }^{1}$, S. Fitoz², N. Numanoglu ${ }^{1}$
}

ABSTRACT: Pulmonary tuberculosis with multifocal skeletal involvement. A. Kaya, Z. Topu, S. Fitoz, N. Numanoglu.

Skeletal tuberculosis accounts for $1-5 \%$ of all tuberculosis infections. Of these infections, $50 \%$ involve the vertebral column and only $0-5 \%$ involve ribs. Rib tuberculosis is seen in only $0.1 \%$ of all tuberculosis infections. Skele- tal tuberculosis is localised to a single site in $90-95 \%$ of reported cases. Multifocal involvement is uncommon and is usually associated with disseminated disease. We present a case of pulmonary tuberculosis associated with multifocal skeletal involvement.

Monaldi Arch Chest Dis 2004; 61: 2, 133-135.

Keywords: Multiple lytic vertebral lesions, Skeletal tuberculosis.

${ }^{1}$ Department of Chest Diseases, Faculty of Medicine, Ankara University, Ankara, Turkey.

2 Department of Radiology, Faculty of Medicine, Ankara University, Ankara, Turkey.

Correspondence: Akın Kaya; Ankara Universitesi TIP Fakultesi; Gogus Hastalıklarl; Dikimevi-Ankara/Turkey; e-mail.akaya@ medicine.ankara.edu.tr

\section{Introduction}

Tuberculosis $(\mathrm{TB})$ is an ancient disease that has long been a major public health challenge. In the 19th century, TB was responsible for nearly one in 10 deaths in Europe [1]. The disease is now increasing in frequency in the developed world due to increased world migration and AIDS epidemics. In addition to classical pulmonary TB, atypical presentation is becoming more common [2]. Skeletal tuberculosis accounts for $1-5 \%$ of all tuberculosis infections. Of these infections, $50 \%$ involve the vertebral column and only $0-5 \%$ involve ribs [3]. Rib tuberculosis is seen in only $0.1 \%$ of all tuberculosis infections [4]. Skeletal tuberculosis is localised to a single site in $90-95 \%$ of reported cases. Multifocal involvement is uncommon and usually associated with disseminated disease [5]. We present a case of pulmonary tuberculosis associated with multifocal skeletal involvement.

\section{Case report}

A 25-year-old male was admitted to the clinic with complaining of a cough, night sweats and pains in his left shoulder, anterolateral and posterolateral chest, left hip and a head-ache, which was exacerbated by general movement since three months. The cough and chest pain prompted five visits to the emergency room, and on all occasions he was given analgesics and non-specific antibiotics. The patient had no past history of tuberculosis and denied recent exposure. He denied risk factors for HIV. His physical exam was remarkable for tenderness over his left lateral chest wall, with point tenderness over several ribs. No masses were appreciated. Serum chemistries and hematological profile were within normal limits. Frontal chest radiography demonstrated right hilar enlargement, perihilar infiltration and lytic expansile $8^{\text {th }}$ rib lesion on the left side (fig. 1). Computed tomography (CT) of the chest showed multiple mediastinal and right hilar lymphadenopathies and right upper lobe consolidation (fig. 2). CT examination also revealed multiple lytic vertebral lesions associated with paravertebral soft tissue and left $8^{\text {th }}$ rib involvement (fig. 3, 4). The differential diagnosis included metastatic disease, lymphoma, disseminated fungal or mycobacterium infection and especially multiple myeloma. A bone scan showed areas of increased uptake in the superior aspect of the left scapula, L2- L3 vertebral bodies, the right lateral ileum, left anterior 4 th rib,

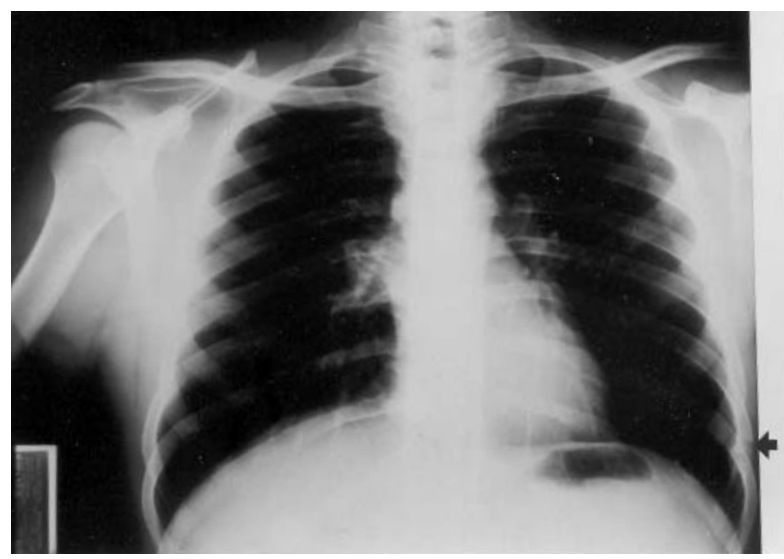

Fig. 1. - Chest-X-ray demonstrates right hilar enlargement and expansile and lytic $8^{\text {th }}$ rib lesion on the left side (arrow). 


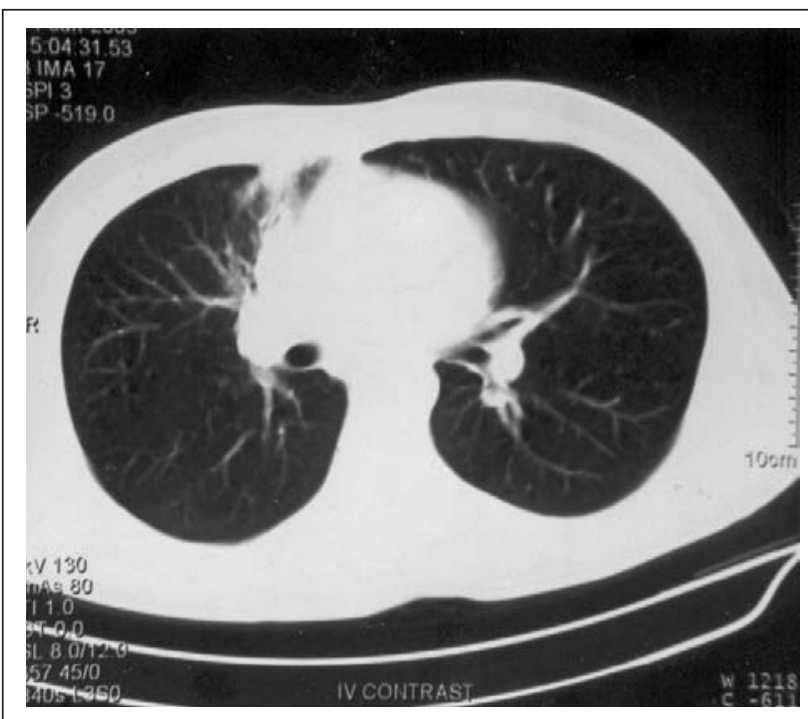

Fig. 2. - Axial thoracic CT shows right upper lobe anterior segment consolidation.

left anterolateral $8^{\text {th }}$ rib, right posterior parietal of cranium, left sternoclavicular joint and bilateral sacroiliac joints (fig. 5). His purified protein derivative (PPD) skin test was positive at $22 \mathrm{~mm}$. Sputum was negative for acid-fast bacilli (AFB). Fiber optic bronchoscopy was normal. No AFB was found in bronchial lavage. Lymph nodes biopsy and a wedge biopsy of the lungs were performed by video-assisted thoracoscopic surgery. The final diagnosis included tuberculosis. Lung biopsy specimens presented small necrotising granulomas with multinucleated giant cells. Identification of acid-fast bacilli and growth of $M y$ cobacterium tuberculosis occurred in lung specimens and specimens from lymph nodes. The patient was started on four anti-tuberculosis drugs (INH, rifampin, pyrazinamide and ethambutol). At 1-month follow-up the patient had complete resolution of symptoms.

\section{Discussion}

Skeletal TB is due mainly to haematogenous dissemination $[3,6])$. TB involvement of ribs may also occur by direct extension from lungs or rupture into lung or pleural space $[3,4,6]$. Active or re-infection type of pulmonary TB is not often seen in association with rib TB[6]. Patients from endemic areas have a higher incidence of multifocal skeletal involvement with radiographic features of periosteal reaction, bone sclerosis, and severe bone destruction at sites such as the rib, pelvis, and cervical spine. Their lesions are often solitary and osteolytic, involving the axial skeleton, thoracolumbar vertebral bodies and hips [7]. In this case of multifocal vertebral TB there was lytic involvement of multiple ribs and other skeletal areas. Between 50 and $75 \%$ of all cases of osteoarticular TB are associated with a primary focus in the lungs [8]. In this case skeletal TB was associated with a primary focus in the lungs and this pattern is consistent with previous reports.
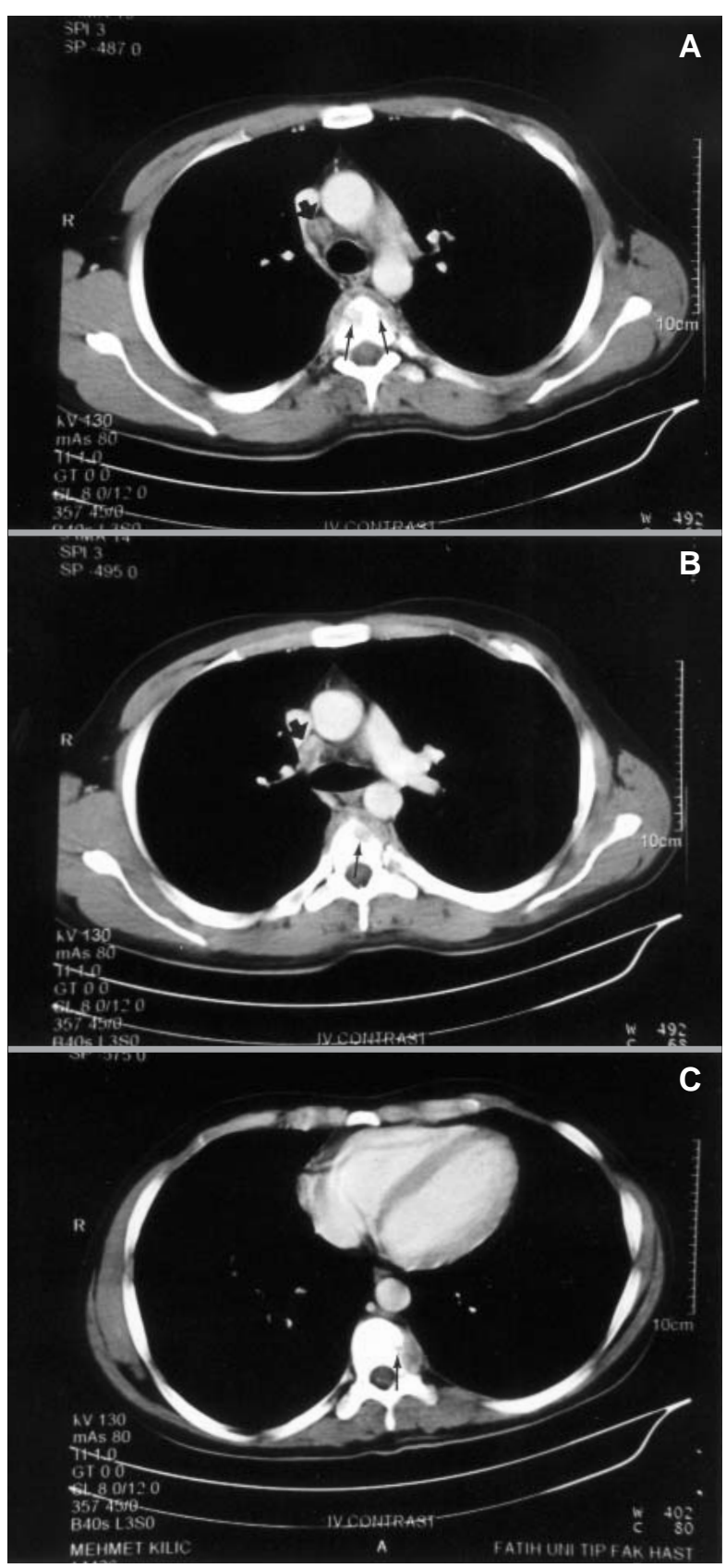

Fig. 3. - CT scans from different levels (A, B, C) demonstrate multiple vertebral hypodense lesions (arrows) and accompanying paravertebral soft tissue. Please note enlarged mediastinal lymph nodes (thick arrows).

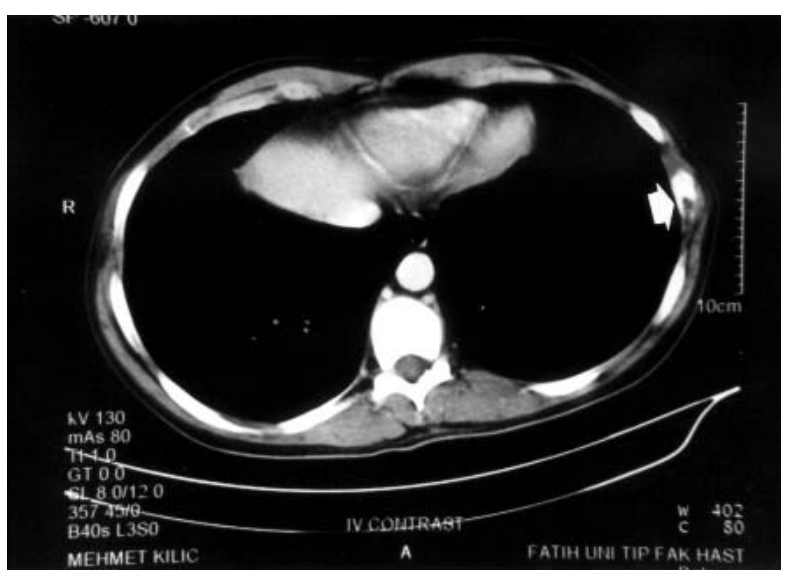

Fig. 4. - CT scan of lung base reveals a lytic expansile costal lesion on the left side (arrow). 


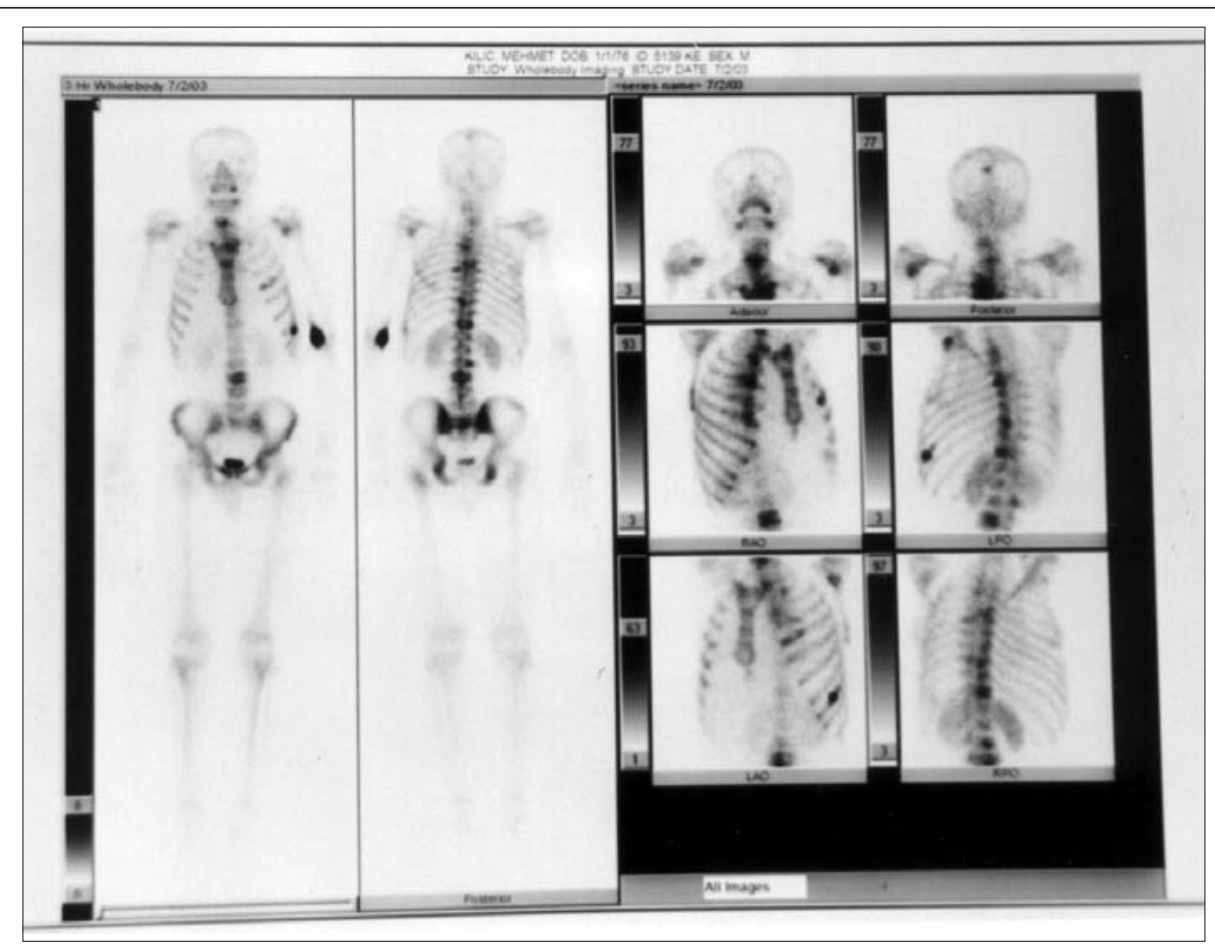

Fig. 5. - A bone scan showed areas of increased uptake in the superior aspect of the left scapula, L2- L3 vertebral bodies, the right lateral ileum, left anterior $4^{\text {th }}$ rib, left anterolateral $8^{\text {th }}$ rib, right posterior parietal of cranium, left sternoclavicular joint and bilateral sacroiliac joints.

The spine is the most common site and accounts for more than $40-60 \%$ of all cases of skeletal TB. The usual locations are lumbar and thoracic. The ribs are involved in only $0-5 \%$ of cases of osteoarticular TB $[9,10,11]$. The rib was the most common bone involved in skeletal TB in heroin-addicted patients [12].

TB of the ribs presents with rib destruction [13]. Clinically, local pain is the most common symptom [14]. This case presented with local pain and pulmonary symptoms.

Radiographic features are predominantly osteolytic. There may be areas of sclerosis $[13,14]$. CT can reveal osteolytic expansile lesions with varying degrees of rib destruction. Also typically found are well-defined juxta-costal soft tissue masses of low attenuation on both sides or on the inner aspect of ribs, with peripheral rim enhancement, the so-called cold abscess [15]. The CT examination in the patient showed an expansile multiloculated lytic lesions with little reactive sclerosis, highly suggestive of metastatic malignancy. In addition, there was evidence of pulmonary involvement on plain radiography or CT of the chest.

TB has seen resurgence recently and there has been a concomitant increase in bone and joint TB. Some attribute this increase to $\operatorname{HIV}[13,14,15]$. However, as demonstrated in our case it can be seen without HIV infection. The diagnosis is frequently delayed in widespread lytic lesions, because this rarely occurs in TB and multiple osteolytic lesions are mistaken for malignancy. The majority of patients with similar lesions are found to have metastatic disease from some primary lesion [16].

The key to diagnosis is to bear in mind that TB can present in multiple unusual sites, especially in immigrants from countries where $\mathrm{TB}$ is endemic.

\section{References}

1. Conolly MA, Chaulet P, Raviglione MC. Epidemiology of tuberculosis. In: R. Wilson. Tuberculosis. Belgium: European Respiratory Society, 1972 (4): pp. 51-67.

2. Arslan A, Çiftçi E, Yıldız F, et al. Multifocal bone tuberculosis presenting as a breast mass: CT and MRI findings. Eur Radiol 1999; 9: 1117-1119.

3. Ip M, Chen NK, So SY, et al. Unusual rib destruction in pleuropulmonary tuberculosis. Chest 1989; 95: 242-244.

4. Tatelman M, Drouillard EJP. Tuberculosis of the ribs. AJR 1953; 70: 923-935.

5. Shannon B, Moore M, Houkon J, et al. Multifocal cystic tuberculosis of bone. J Bone Joint Surgery Br 1990; 72: 1089-1092.

6. Adler BD, Padley SPG, Müller N. Tuberculosis of the chest wall: CT findings. J Comput Assist Tomogr 1993; 17: 271-273.

7. Alvarez S, McCabe WR. Extrapulmonary tuberculosis revisited: a review of experience at Boston City and other hospitals. Medicine 1984; 63: 25-55.

8. Ediken J, Hodes PJ. Roentgen diagnosis of diseases of bone. Baltimore: Williams and Wilkins, 1973.

9. Weaver P, Lifeso RM. The radiological diagnosis of tuberculosis of the adult spine. Skeletal Radiol 1984; 12: 178-186.

10. Davies PD, Humphries MJ, Byfield SP, et al. Bone and joint tuberculosis - a survey of notification in England and Wales. J Bone Joint Surg Br 1984; 66: 326-330.

11. Wolstein D, Rabinowitz J, Twersky J. Tuberculosis of the rib. J Can Assoc Radiol 1974; 25: 307-309.

12. Firooznia H, Seliger G, Abrams RM, et al. Disseminated extrapulmonary tuberculosis in association with heroin addiction. Radiology 1973; 109: 291-296.

13. Wiebe ER, Elwood RK. Tuberculosis of the ribs - a report of three cases. Respir Med 1991; 85: 251-253.

14. Lee $\mathrm{S}$, Abramson S. Infections of the musculoskeletal system by M. Tuberculosis. In: Rom W, Gray S (eds) Tuberculosis Boston: Little Brown, 1996: pp. 635-644.

15. Lee G, Im JG, Kim JS, et al. Tuberculosis of the ribs: CT appearance. J Comput Assist Tomogr 1993; 17: 363-366.

16. Chang DS, Rafii M, McGuinnes G, et al. Primary multifocal tuberculous osteomyelitis with involvement of the ribs. Skeletal Radiol 1998; 27: 641-645. 